\title{
Positive Solutions for a Class of Fourth-Order $p$-Laplacian Boundary Value Problem Involving Integral Conditions
}

\author{
Yan Sun \\ Department of Mathematics, Shanghai Normal University, Shanghai 200234, China \\ Correspondence should be addressed to Yan Sun; ysun@shnu.edu.cn
}

Received 30 April 2015; Revised 19 July 2015; Accepted 27 July 2015

Academic Editor: Josef Diblík

Copyright (C) 2015 Yan Sun. This is an open access article distributed under the Creative Commons Attribution License, which permits unrestricted use, distribution, and reproduction in any medium, provided the original work is properly cited.

Under some conditions concerning the first eigenvalues corresponding to the relevant linear operator, we obtain sharp optimal criteria for the existence of positive solutions for $p$-Laplacian problems with integral boundary conditions. The main methods in the paper are constructing an available integral operator and combining fixed point index theory. The interesting point of the results is that the nonlinear term contains all lower-order derivatives explicitly. Finally, we give some examples to demonstrate the main results.

\section{Introduction}

In this paper, we discuss the existence of positive solutions for the following $p$-Laplacian integral boundary value problems:

$$
\begin{aligned}
& \left(\phi_{p}\left(y^{\prime \prime}(t)\right)\right)^{\prime \prime} \\
& =a(t) f\left(t, y(t), y^{\prime}(t), y^{\prime \prime}(t), y^{\prime \prime \prime}(t)\right), \\
& \quad 0<t<1, \\
& \begin{array}{l}
y(0)=y(1)=\int_{0}^{1} b(s) y(s) \mathrm{d} s, \\
y^{\prime \prime}(0)=y^{\prime \prime}(1)=\phi_{q}\left(\int_{0}^{1} c(s) \phi_{p}\left(y^{\prime \prime}(s)\right) \mathrm{d} s\right),
\end{array}
\end{aligned}
$$

where $\phi_{p}(t)=|t|^{p-2} t, p>1, \phi_{q}=\phi_{p}^{-1}, 1 / p+1 / q=1, a \epsilon$ $L^{1}[0,1]$ is symmetric on the interval $[0,1]$ (i.e., $a(1-t)=a(t)$ and $a(t) \geq 0$ for $t \in[0,1]), f:[0,1] \times[0,+\infty) \times(-\infty,+\infty) \times$ $(-\infty,+\infty) \times(-\infty,+\infty) \rightarrow[0,+\infty)$ is continuous, and $b, c \in$ $L^{1}[0,1]$ are nonnegative symmetric on $[0,1]$.

Boundary value problems of ordinary differential equations have become an important research field in recent years. Fourth-order $p$-Laplacian boundary value problems arise in applied mathematics, physics, gas diffusion through porous media, engineering, elastic mechanics, electromagnetic waves of gravity driven flows, and the various areas of adiabatic tubular reactor processes, as well as biological problems; see [1-5] and the references therein.

In [6], by using the fixed point theorem for strict set contraction operator, Zhang et al. considered the existence of positive solutions for the following $n$ th-order impulsive boundary value problems with integral boundary conditions in Banach spaces:

$$
\begin{aligned}
& z^{(n)}(t)+g\left(t, z(t), z^{\prime}(t), \ldots, z^{(n-2)}(t)\right)=\theta, \\
& t \in J, t \neq t_{k}, \quad k=1, \ldots, m, \\
& \left.\Delta z^{(n-1)}\right|_{t=t_{k}}=-I_{k}\left(z^{(n-2)}\left(t_{k}\right)\right), \quad k=1, \ldots, m, \\
& z^{(i)}(0)=\theta, \quad i=0,1, \ldots, n-3, \\
& z^{(n-2)}(0)=z^{(n-2)}(1)=\int_{0}^{1} h(t) z^{(n-2)}(t) \mathrm{d} t,
\end{aligned}
$$

where $J=[0,1], 0<t_{1}<t_{2}<\cdots<t_{k}<\cdots<t_{m}<1$, $g \in C\left[J \times P^{n-1}, P\right], I_{k} \in C[P, P], k=1, \ldots, m, h \in L^{1}[0,1]$, and $\left.\Delta z^{(n-1)}\right|_{t=t_{k}}=z^{(n-1)}\left(t_{k}^{+}\right)-z^{(n-1)}\left(t_{k}^{-}\right)$denotes the jump of $z^{(n-1)}(t)$ at $t=t_{k}$. They also gave the upper and lower bounds for these positive solutions. 
In [7], by means of the fixed point index theory, Sun and Zhang studied the following singular nonlinear Sturm-Liouville problems:

$$
\begin{aligned}
-(L \phi)(x) & =a(x) g(\phi(x)), \quad 0<x<1, \\
R_{1}(\phi) & =\alpha_{1} \phi(0)+\beta_{1} \phi^{\prime}(0)=0, \\
R_{2}(\phi) & =\alpha_{2} \phi(1)+\beta_{2} \phi^{\prime}(1)=0, \\
-(L \phi)(x) & =f(x, \phi(x)), \quad 0<x<1, \\
R_{1}(\phi) & =\alpha_{1} \phi(0)+\beta_{1} p(x) \phi^{\prime}(0)=0, \\
R_{2}(\phi) & =\alpha_{2} \phi(1)+\beta_{2} p(x) \phi^{\prime}(1)=0,
\end{aligned}
$$

where $(L \phi)(x)=\left(p(x) \phi^{\prime}(x)\right)^{\prime}+q(x) \phi(x)$ and $a(x)$ is allowed to be singular at $x=0$ and $x=1$.

In [2], by making use of the theory of the spectrum, Sergejeva studied the regions of solvability for three-point boundary value problem:

$$
\begin{aligned}
-y^{\prime \prime}(t) & =\mu y^{+}-\lambda y^{-}+h\left(t, y, y^{\prime \prime}\right), \quad t \in(0,1), \\
y(0) & =0 \\
y(1) & =c y\left(\frac{1}{2}\right)
\end{aligned}
$$

where $y^{+}=\max \{y, 0\}, y^{-}=\max \{-y, 0\}$, and $h$ is a bounded function. The solvability results are established for the problem with $h \equiv 0$.

Motivated and inspired greatly by the above mentioned works, the aim of the paper is to improve, generalize, and supplement the previous results. We obtain the existence results on nonlinear fourth-order integral boundary value problems with one-dimensional $p$-Laplacian integral boundary value problem (1). The novelty of our results is in exploring some optimal criteria for the existence of positive solutions of problem (1). The methods used in our work will depend on an application of fixed point index theory together with the first eigenvalues corresponding to the relevant linear operator. It can also be seen that the nonlinear term involves all lowerorder derivatives explicitly.

The paper is organized as follows. In Section 2, we state some lemmas and several preliminary results. The main results are formulated and proved in Section 3. Examples are given in Section 4.

\section{Preliminaries}

Throughout the paper, we make the following assumptions:

$\left(\mathbf{H}_{1}\right) f \in C([0,1] \times[0,+\infty) \times(-\infty,+\infty) \times(-\infty,+\infty) \times$ $(-\infty,+\infty),[0,+\infty))$ and $h(t, y) \leq f\left(t, y, y^{\prime}, y^{\prime \prime}, y^{\prime \prime \prime}\right) \leq g(t$, $y), h, g:[0,1] \times[0,+\infty) \rightarrow[0,+\infty)$, are continuous and also $h(\cdot, y)$ and $g(\cdot, y)$ are symmetric on $[0,1]$ for all $y \geq 0$ (i.e., $h(1-t, y)=h(t, y), g(1-t, y)=g(t, y))$.

$\left(\mathbf{H}_{2}\right) a, b, c \in L^{1}[0,1]$ are all nonnegative and symmetric on $[0,1]$, and $a(t) \neq \equiv$ is continuous on $(0,1)$, and $a(t)$ may be singular at $t=0$ and/or $t=1$, and $\mu \in(0,1)$ and $\nu \in(0,1)$, where

$$
\begin{aligned}
& \mu=\int_{0}^{1} b(s) \mathrm{d} s, \\
& \nu=\int_{0}^{1} c(s) \mathrm{d} s .
\end{aligned}
$$

In what follows, we will consider the Banach space $E=$ $C[0,1], C^{+}[0,1]=\{x \in C[0,1] \mid x(t) \geq 0\}$, equipped with the maximum norm $\|z\|=\max _{0 \leq t \leq 1}|z(t)|$. Denote cone $P$ by

$$
P=\left\{z \in C^{+}[0,1] \mid z(t) \text { is concave for } t \in[0,1]\right\} ;
$$

then, $P$ is a positive cone in $C[0,1]$.

Let $B_{r}=\left\{z \in C^{+}[0,1] \mid\|z\|<r\right\}$ for $r(r>0)$, and

$$
\begin{gathered}
H(t, s)=G(t, s)+\frac{1}{1-\mu} \int_{0}^{1} G(s, \tau) b(\tau) \mathrm{d} \tau, \\
H_{1}(t, s)=G(t, s)+\frac{1}{1-v} \int_{0}^{1} G(s, v) c(v) \mathrm{d} v, \\
G(t, s)= \begin{cases}s(1-t), & 0 \leq s \leq t \leq 1, \\
t(1-s), & 0 \leq t \leq s \leq 1 .\end{cases}
\end{gathered}
$$

Here $G(t, s), H(t, s)$, and $H_{1}(t, s)$ are Green's functions of problem (1).

Lemma 1 (see [1]). Suppose that $\left(\boldsymbol{H}_{1}\right)$ and $\left(\boldsymbol{H}_{2}\right)$ hold; then, for $t, s \in[0,1]$, we have

(I) $G(t, s) \geq 0, H(t, s) \geq 0, H_{1}(t, s) \geq 0$;

(II) $e(t) e(s) \leq G(t, s) \leq e(t) \leq 1 / 4$, where $e(t)=t(1-t)$;

(III) $G(1-t, 1-s)=G(t, s), H(1-t, 1-s)=H(t, s)$, and $H_{1}(1-t, 1-s)=H_{1}(t, s)$;

(IV) $\rho e(s) \leq H(t, s) \leq \gamma s(1-s)=\gamma e(s) \leq(1 / 4) \gamma ; \rho_{1} e(s) \leq$ $H_{1}(t, s) \leq \gamma_{1} s(1-s)=\gamma_{1} e(s) \leq(1 / 4) \gamma_{1}$, where $\gamma=$ $1 /(1-\mu), \rho=\int_{0}^{1} e(\tau) b(\tau) \mathrm{d} \tau /(1-\mu), \gamma_{1}=1 /(1-\nu)$, $\rho_{1}=\int_{0}^{1} e(\tau) c(\tau) \mathrm{d} \tau /(1-\nu)$.

Define an integral operator $A: C^{+}[0,1] \rightarrow C^{+}[0,1]$ by

$$
\begin{aligned}
& (A y)(t)=\int_{0}^{1} H(t, s) \phi_{q}\left[\int_{0}^{1} H_{1}(s, \tau) a(\tau)\right. \\
& \left.\cdot f\left(\tau, y(\tau), y^{\prime}(\tau), y^{\prime \prime}(\tau), y^{\prime \prime \prime}(\tau)\right) \mathrm{d} \tau\right] \mathrm{d} s .
\end{aligned}
$$

It is well known that $y \in C^{+}[0,1]$ is a solution of problem (1) if and only if $y$ is a fixed point of operator equation $y=A y$.

Lemma 2. Suppose that $\left(\boldsymbol{H}_{1}\right)$ and $\left(\boldsymbol{H}_{2}\right)$ hold, if $y \in E$ is a solution of the following integral equation:

$$
\begin{aligned}
& y(t)=\int_{0}^{1} H(t, s) \phi_{q}\left[\int_{0}^{1} H_{1}(s, \tau) a(\tau)\right. \\
& \left.\cdot f\left(\tau, y(\tau), y^{\prime}(\tau), y^{\prime \prime}(\tau), y^{\prime \prime \prime}(\tau)\right) \mathrm{d} \tau\right] \mathrm{d} s
\end{aligned}
$$

then, $y$ is a solution of problem (1). 
Lemma 3. Suppose that $\left(\boldsymbol{H}_{1}\right)$ and $\left(\boldsymbol{H}_{2}\right)$ hold; then, $A: P \rightarrow P$ is a completely continuous operator.

Proof. First, we prove that $A: P \rightarrow P$. For every $y \in P$, we have

$$
\begin{aligned}
& (A y)^{\prime \prime}(t)=-\phi_{q}\left[\int_{0}^{1} H_{1}(t, s) a(s)\right. \\
& \left.\cdot f\left(s, y(s), y^{\prime}(s), y^{\prime \prime}(s), y^{\prime \prime \prime}(s)\right) \mathrm{d} s\right] \leq 0, \\
& (A y)(0)=\int_{0}^{1} H(0, s) \phi_{q}\left[\int_{0}^{1} H_{1}(s, \tau) a(\tau)\right. \\
& \left.\cdot f\left(\tau, y(\tau), y^{\prime}(\tau), y^{\prime \prime}(\tau), y^{\prime \prime \prime}(\tau)\right) \mathrm{d} \tau\right] \mathrm{d} s \\
& \geq 0
\end{aligned}
$$$$
(A y)(1)=\int_{0}^{1} H(1, s) \phi_{q}\left[\int_{0}^{1} H_{1}(s, \tau) a(\tau)\right.
$$$$
\left.f\left(\tau, y(\tau), y^{\prime}(\tau), y^{\prime \prime}(\tau), y^{\prime \prime \prime}(\tau)\right) \mathrm{d} \tau\right] \mathrm{d} s
$$$$
\geq 0 \text {. }
$$

Thus, $(A y)(t) \geq 0$, for all $t \in[0,1]$. Hence, $(A y)(t)$ is nonnegative concave functional on $[0,1]$.

By Lemma 1 , for $t \in[0,1]$, we obtain

$$
\begin{aligned}
& (A y)(t)=\int_{0}^{1} H(t, s) \phi_{q}\left[\int_{0}^{1} H_{1}(s, \tau) a(\tau)\right. \\
& \left.\cdot f\left(\tau, y(\tau), y^{\prime}(\tau), y^{\prime \prime}(\tau), y^{\prime \prime \prime}(\tau)\right) \mathrm{d} \tau\right] \mathrm{d} s \\
& \leq \int_{0}^{1} H(t, s) \phi_{q}\left[\int_{0}^{1} H_{1}(s, \tau) a(\tau)\right. \\
& \cdot g(\tau, y(\tau)) \mathrm{d} \tau] \mathrm{d} s \leq \gamma \gamma_{1}^{q-1} \int_{0}^{1} e(s) \\
& \cdot \phi_{q}\left[\int_{0}^{1} e(\tau) a(\tau) g(\tau, y(\tau)) \mathrm{d} \tau\right] \mathrm{d} s<+\infty .
\end{aligned}
$$

Hence, $A y \in P$, and $A(P) \subset P$, which implies $A: P \rightarrow P$.

Next, we show that $A: P \rightarrow P$ is completely continuous.

For any natural number $n \geq 2$, set

$$
a_{n}(t)= \begin{cases}\inf _{t \leq s \leq 1 / n} a(s), & 0 \leq t \leq \frac{1}{n} \\ a(t), & \frac{1}{n} \leq t \leq \frac{n-1}{n}, \\ \inf _{(n-1) / n \leq s \leq t} a(s), & \frac{n-1}{n} \leq t \leq 1 .\end{cases}
$$

Then, $a_{n}:[0,1] \rightarrow[0,+\infty)$ is continuous, and $a_{n}(t) \leq a(t)$, $t \in[0,1]$. Let

$$
\begin{gathered}
\left(A_{n} y\right)(t)=\int_{0}^{1} H(t, s) \phi_{q}\left[\int_{0}^{1} H_{1}(s, \tau) a_{n}(\tau)\right. \\
\left.\cdot f\left(\tau, y(\tau), y^{\prime}(\tau), y^{\prime \prime}(\tau), y^{\prime \prime \prime}(\tau)\right) \mathrm{d} \tau\right] \mathrm{d} s .
\end{gathered}
$$

Obviously, $A_{n}: P \rightarrow P$ is a completely continuous operator. From Lemma 1, we obtain

$$
\begin{aligned}
& \lim _{n \rightarrow \infty}\left\|A_{n} y-A y\right\| \leq \lim _{n \rightarrow \infty} \max _{0 \leq t \leq 1} \int_{0}^{1} H(t, s) \\
& \cdot \phi_{q}\left[\int_{0}^{1} H_{1}(s, \tau)\left(a(\tau)-a_{n}(\tau)\right) g(\tau, y(\tau)) \mathrm{d} \tau\right] \mathrm{d} s \\
& \leq \gamma \gamma_{1}^{q-1} \lim _{n \rightarrow \infty} \int_{0}^{1} e(s) \\
& \cdot \phi_{q}\left[\int_{0}^{1} e(\tau) a(\tau) g(\tau, y(\tau)) \mathrm{d} \tau\right] \mathrm{d} s=\gamma \gamma_{1}^{q-1} \\
& \cdot \lim _{n \rightarrow \infty} \int_{e(n)} e(s) \phi_{q}\left[\int_{0}^{1} e(\tau) a(\tau) g(\tau, y(\tau)) \mathrm{d} \tau\right] \mathrm{d} s \\
& \quad=0,
\end{aligned}
$$

where $e(n)=[0,1 / n] \cup[(n-1) / n, 1]$. Therefore, $A: P \rightarrow P$ is a completely continuous operator. The proof is completed.

Now we define linear integral operator as follows:

$$
\begin{aligned}
& (T y)(t) \\
& \quad=\int_{0}^{1} H(t, s) \phi_{q}\left[\int_{0}^{1} H_{1}(s, \tau) a(\tau) y(\tau) \mathrm{d} \tau\right] \mathrm{d} s, \\
& L=\max _{t \in[0,1]} \int_{0}^{1} H(t, s) \phi_{q}\left[\int_{0}^{1} H_{1}(s, \tau) a(\tau) \mathrm{d} \tau\right] \mathrm{d} s .
\end{aligned}
$$

It is easy to see that $T: C^{+}[0,1] \rightarrow C^{+}[0,1]$ is a completely continuous linear operator, and $T(P) \subset P$.

Lemma 4. Assume that $\left(\boldsymbol{H}_{1}\right)$ and $\left(\boldsymbol{H}_{2}\right)$ hold. Then, the operator $T: P \rightarrow P$ defined by (15) is a completely continuous linear operator and $T(P) \subset P$, the spectral radius $r(T) \neq 0$, and $T$ has a positive eigenfunction corresponding to its first eigenvalue $\lambda_{1}=r^{-1}(T)$.

Proof. A simple modification of the argument in Lemma 3 yields that $T: P \rightarrow P$ is a completely continuous linear operator and $T(P) \subset P$. By $\left(\mathbf{H}_{1}\right)$ and $\left(\mathbf{H}_{2}\right)$, there is $s_{0} \in(0,1)$ such that $H_{1}\left(s_{0}, s_{0}\right) a\left(s_{0}\right)>0$. Choose constants $u$ and $v$ such that $s_{0} \in(u, v) \subset[u, v] \subset(0,1)$ and $H_{1}(s, s) a(s)>0$, for all $t, s \in[u, v]$. Choose a nonnegative continuous functional 
$g \in C[0,1]$ such that $g(t)>0$, for all $t \in(u, v)$. Then, for any $t \in(u, v)$, we have

$$
\begin{aligned}
& (\mathrm{Tg})(t) \\
& =\int_{0}^{1} H(t, s) \phi_{q}\left[\int_{0}^{1} H_{1}(s, \tau) a(\tau) g(\tau) \mathrm{d} \tau\right] \mathrm{d} s \\
& \quad \geq \int_{u}^{v} H(t, s) \phi_{q}\left[\int_{u}^{v} H_{1}(s, \tau) a(\tau) g(\tau) \mathrm{d} \tau\right] \mathrm{d} s \\
& \geq 0 .
\end{aligned}
$$

Thus, there exists a constant $w>0$ such that $w(\mathrm{Tg})(t) \geq$ $g(t)$, for all $t \in[0,1]$. By famous Krein-Rutman theorems, we know that the spectral radius $r(T) \neq 0$ and $T$ has a positive eigenfunction $\psi$ corresponding to its first eigenvalue $\lambda_{1}=r^{-1}(T)$; that is, $\psi=\lambda_{1} T \psi$. This completes the proof.

Lemma 5 (see [8]). Let $P$ be a cone in Banach space E, and let $\Omega(P)$ be a bounded open set in $P$. Suppose that $A: \bar{\Omega}(P) \rightarrow P$ is completely continuous. If there exists $y_{0} \in P \backslash\{\theta\}$, such that $y-A y \neq \mu y_{0}$, for all $y \in \partial \Omega(P), \mu \geq 0$. Then, the fixed point index $i(A, \Omega(P), P)=0$.

Lemma 6 (see [8]). Let $P$ be a cone in Banach space E, and let $\Omega(P)$ be a bounded open set in $P$ with $\theta \in \Omega(P)$. Suppose that $A: \bar{\Omega}(P) \rightarrow P$ is a completely continuous operator. If $A y \neq \mu y$, for all $y \in \partial \Omega(P), \mu \geq 1$. Then, the fixed point index $i(A, \Omega(P), P)=1$.

Lemma 7 (see [9]). Let $P$ and $P_{1}$ be cones in Banach space $E$, and let $\Omega(P)$ be a bounded open set in $P$. Suppose that $A$ : $\bar{\Omega}(P) \rightarrow P$ is a completely continuous operator, and $A$ has no fixed point on $\partial \Omega(P)$. If there exist linear operators $L_{1}, L_{2}, L_{3}$ : $E \rightarrow E$ which satisfy $L_{1}(P) \subset P, L_{2}(P) \subset P_{1}, L_{3}\left(P_{1}\right) \subset P_{1}$, and $y_{0} \in P \backslash\{\theta\}$, such that

(1) $L_{2} L_{1}^{n} y_{0} \geq L_{2} y_{0}$, for certain natural number $n$,

(2) $L_{2} L_{1} y=L_{3} L_{2} y$, for all $y \in P$,

(3) $L_{2} A y \geq L_{2} L_{1} y$, for all $y \in \partial \Omega(P)$,

then the fixed point index $i(A, \Omega(P), P)=0$.

\section{Main Results}

In this section, we establish sharp optimal criteria for the existence of positive solutions to problem (1) under superlinear cases and sublinear cases, respectively.

Theorem 8. Suppose that $\left(\boldsymbol{H}_{1}\right)$ and $\left(\boldsymbol{H}_{2}\right)$ hold. In addition, assume that

$$
\begin{gathered}
\liminf _{y \rightarrow+\infty} \frac{h(t, y(t))}{y}>\lambda_{1}, \quad \text { uniformly on } t \in[0,1], \\
\underset{y \rightarrow 0+}{\limsup } \frac{g(t, y(t))}{y}<\lambda_{1}, \quad \text { uniformly on } t \in[0,1]
\end{gathered}
$$

where $\lambda_{1}$ is the first eigenvalue of $T$ defined by (15); then, problem (1) has at least one positive solution.
To prove our main result, we need some preliminary results.

Lemma 9. Suppose that $\left(\boldsymbol{H}_{1}\right)$ and $\left(\boldsymbol{H}_{2}\right)$ hold. Define

$$
P_{1}=\left\{y \in P \mid \int_{0}^{1} \tilde{y}^{*}(t) y(t) \mathrm{d} t \geq \lambda_{1}^{-1} \delta\|y\|\right\},
$$

where $\tilde{y}^{*}(t)=y^{*}(t) a(t), y^{*} \in P$, is the positive eigenfunction corresponding to its first eigenvalue $\lambda_{1}=r^{-1}(T)$; that is, $y^{*}=$ $\lambda_{1} T y^{*}$, and

$$
\delta \leq \frac{\int_{0}^{1} y^{*}(t) a(t) y(t) \mathrm{d} t}{\int_{0}^{1} H(t, s) \phi_{q}\left[\int_{0}^{1} H_{1}(s, \tau) a(\tau) y(\tau) \mathrm{d} \tau\right] \mathrm{d} s}
$$

then, $P_{1}$ is a cone in $C^{+}[0,1]$, and $T(P) \subset P_{1}$.

Proof. It is obvious that $P_{1}$ is a cone in $C^{+}[0,1]$. For any $y \in P$, we obtain

$$
\begin{aligned}
& \int_{0}^{1} \tilde{y}^{*}(t)(T y)(t) \mathrm{d} t=\int_{0}^{1} \tilde{y}^{*}(t) \mathrm{d} t \\
& \quad \int_{0}^{1} H(t, s) \phi_{q}\left[\int_{0}^{1} H_{1}(s, \tau) a(\tau) y(\tau) \mathrm{d} \tau\right] \mathrm{d} s \\
& =\lambda_{1}^{-1} \int_{0}^{1} \tilde{y}^{*}(t) y(t) \mathrm{d} t \\
& =\lambda_{1}^{-1} \int_{0}^{1} y^{*}(t) a(t) y(t) \mathrm{d} t \geq \lambda_{1}^{-1} \delta\|T y\|
\end{aligned}
$$

Thus, $T(P) \subset P_{1}$. The proof is completed.

Proof of Theorem 8. It follows from (17) that there exists $\varepsilon>0$, such that $h(t, y(t)) \geq\left(\lambda_{1}+\varepsilon\right) y$, for $y$ is sufficiently large. In view of $\left(\mathbf{H}_{1}\right)$, we see that there exists $b \geq 0$ such that

$$
h(t, y(t)) \geq\left(\lambda_{1}+\varepsilon\right) y-b, \quad 0 \leq y<+\infty
$$

Let $R>L b \lambda_{1}^{2}(\varepsilon \delta)^{-1} \int_{0}^{1} \tilde{y}^{*}(t) \mathrm{d} t$, where $\widetilde{y}^{*}$ and $\delta$ are defined by Lemma 9.

In the following, we prove that

$$
y-A y \neq \mu y^{*}, \quad \forall y \in \partial B_{R} \cap P, \mu \geq 0,
$$

where $y^{*} \in P$ is a positive eigenfunction corresponding to its first eigenvalue $\lambda_{1}=r^{-1}(T)$.

Otherwise, there exist $y_{1} \in \partial B_{R} \cap P$ and $\mu_{0} \geq 0$, such that

$$
y_{1}-A y_{1}=\mu_{0} y^{*}
$$


Since $A(P) \subset P_{1}$ and $T(P) \subset P_{1}$, we know from (24) that $y_{1} \in P_{1}$. Therefore, it follows from (19) and (22) that

$$
\begin{aligned}
& \int_{0}^{1} \tilde{y}^{*}(t)\left(A y_{1}\right)(t) \mathrm{d} t-\int_{0}^{1} \tilde{y}^{*}(t) y_{1}(t) \mathrm{d} t \geq \int_{0}^{1} \mathrm{~d} t \\
& \cdot \int_{0}^{1} \tilde{y}^{*}(t) H(t, s) \\
& \cdot \phi_{q}\left[\int_{0}^{1} H_{1}(s, \tau) a(\tau) h\left(\tau, y_{1}(\tau)\right) \mathrm{d} \tau\right] \mathrm{d} s \\
& -\int_{0}^{1} \tilde{y}^{*}(t) y_{1}(t) \mathrm{d} t \geq(\lambda,+\varepsilon) \int_{0}^{1} \mathrm{~d} t \int_{0}^{1} \tilde{y}^{*}(t) \\
& \cdot H(t, s) \phi_{q}\left[\int_{0}^{1} H_{1}(s, \tau) a(\tau) y_{1}(\tau) \mathrm{d} \tau\right] \mathrm{d} s \\
& -b \int_{0}^{1} \mathrm{~d} t \int_{0}^{1} \tilde{y}^{*}(t) H(t, s) \\
& -b L \int_{0}^{1} \tilde{y}^{*}(t) \mathrm{d} t>0 . \\
& =\varepsilon \lambda_{1}^{-1} \int_{0}^{1} \tilde{y}_{0}^{*}(t) y_{1}(t) \mathrm{d} t-b L \int_{0}^{1} \tilde{y}^{*}(t) \mathrm{d} t \\
& -y_{1}(t) \mathrm{d} t=\left(\lambda L \int_{0}^{1} \tilde{y}^{*}(t) \mathrm{d} t-\int_{0}^{1} \tilde{y}^{*}(t) y_{1}(t) \mathrm{d} t\right. \\
& \quad(\tau) \mathrm{d} \tau] \mathrm{d} s-\int_{0}^{1} \tilde{y}^{*}(t)
\end{aligned}
$$

On the other hand, it follows from (24) that

$$
\begin{gathered}
\int_{0}^{1} \tilde{y}^{*}(t) y_{1}(t) \mathrm{d} t-\int_{0}^{1} \tilde{y}^{*}(t)\left(A y_{1}\right)(t) \mathrm{d} t \\
\quad=\mu_{0} \int_{0}^{1} \tilde{y}^{*}(t) y^{*}(t) \mathrm{d} t \geq 0,
\end{gathered}
$$

which is a contradiction; thus, by Lemma 5 , we see

$$
i\left(A, B_{R} \cap P, P\right)=0 .
$$

It follows from (18) that there exists $0 \leq r \leq R$, such that

$$
g(t, y(t))<\lambda_{1} y, \quad \forall 0 \leq y \leq r .
$$

Suppose that there exist $y_{2} \in \partial B_{r} \cap P, \mu_{1} \geq 1$, such that $A y_{2}=$ $\mu_{1} y_{2}$. Without loss of generality, we may suppose that $\mu_{1}>1$ (otherwise, the proof is completed). Then,

$$
\begin{aligned}
& \mu_{1} y_{2}(t) \leq \int_{0}^{1} H(t, s) \\
& \cdot \phi_{q}\left[\int_{0}^{1} H_{1}(s, \tau) a(\tau) g\left(\tau, y_{2}(\tau)\right) \mathrm{d} \tau\right] \mathrm{d} s \\
& <\lambda_{1} \int_{0}^{1} H(t, s) \\
& \cdot \phi_{q}\left[\int_{0}^{1} H_{1}(s, \tau) a(\tau) y_{2}(\tau) \mathrm{d} \tau\right] \mathrm{d} s .
\end{aligned}
$$

Multiplying $\tilde{y}^{*}$ and then integrating by (29), we have

$$
\begin{aligned}
& \mu_{1} \int_{0}^{1} \tilde{y}^{*}(t) y_{2}(t) \mathrm{d} t<\lambda_{1} \int_{0}^{1} \mathrm{~d} t \int_{0}^{1} \tilde{y}^{*}(t) H(t, s) \\
& \cdot \phi_{q}\left[\int_{0}^{1} H_{1}(s, \tau) a(\tau) y_{2}(\tau) \mathrm{d} \tau\right] \mathrm{d} s=\lambda_{1} \int_{0}^{1} \mathrm{~d} t \\
& \cdot \int_{0}^{1} H(t, s) \tilde{y}^{*}(t) \\
& \cdot \phi_{q}\left[\int_{0}^{1} H_{1}(s, \tau) a(\tau) y_{2}(\tau) \mathrm{d} \tau\right] \mathrm{d} s=\int_{0}^{1} \tilde{y}^{*}(t) \\
& \cdot y_{2}(t) \mathrm{d} t .
\end{aligned}
$$

By maximum principle, $y^{*}(t)>0$ and $y_{2}(t)>0, t \in[0,1]$; then $\int_{0}^{1} \tilde{y}^{*}(t) y_{2}(t) \mathrm{d} t>0$. Hence, (30) implies $\mu_{1}<1$ which is a contradiction. So by Lemma 6 , we have

$$
i\left(A, B_{r} \cap P, P\right)=1 \text {. }
$$

It follows from (27) and (31) that

$$
\begin{aligned}
& i\left(A,\left(B_{R} \cap P\right) \backslash\left(\bar{B}_{r} \cap P\right), P\right) \\
& \quad=i\left(A, B_{R} \cap P, P\right)-i\left(A, B_{r} \cap P, P\right)=0-1=-1 .
\end{aligned}
$$

Then, $A$ has at least one fixed point on $\left(B_{R} \cap P\right) \backslash\left(\bar{B}_{r} \cap P\right)$. This means problem (1) has at least one positive solution. The proof is completed.

Corollary 10. Suppose that $\left(\boldsymbol{H}_{1}\right)$ and $\left(\boldsymbol{H}_{2}\right)$ hold. In addition, assume that

$$
\begin{aligned}
0 & \leq \limsup _{y \rightarrow 0^{+}} \frac{g(t, y(t))}{y}=g^{0}<h_{\infty} \\
& =\liminf _{y \rightarrow+\infty} \frac{h(t, y(t))}{y} \leq+\infty .
\end{aligned}
$$


Then, for any

$$
\xi \in\left(\frac{\lambda_{1}}{h_{\infty}}, \frac{\lambda_{1}}{g^{0}}\right),
$$

where $\lambda_{1}$ is the first eigenvalue of $T$ defined by (15), the following p-Laplacian integral boundary value problem

$$
\begin{aligned}
& \left(\phi_{p}\left(y^{\prime \prime}(t)\right)\right)^{\prime \prime} \\
& =\xi a(t) f\left(t, y(t), y^{\prime}(t), y^{\prime \prime}(t), y^{\prime \prime \prime}(t)\right), \\
& y(0)=y(1)=\int_{0}^{1} b(s) y(s) \mathrm{d} s, \\
& \phi_{p}\left(y^{\prime \prime}(0)\right)=\phi_{p}\left(y^{\prime \prime}(1)\right)=\int_{0}^{1} c(s) \phi_{p}\left(y^{\prime \prime}(s)\right) \mathrm{d} s
\end{aligned}
$$

has at least one positive solution.

Proof. By (34), we obtain

$$
\begin{gathered}
\liminf _{y \rightarrow+\infty} \frac{\xi h(t, y(t))}{y}>\lambda_{1}, \\
\limsup _{y \rightarrow 0^{+}} \frac{\xi g(t, y(t))}{y}<\lambda_{1} .
\end{gathered}
$$

Therefore, it follows from Theorem 8 that Corollary 10 holds.

Theorem 11. Suppose that $\left(\boldsymbol{H}_{1}\right)$ and $\left(\boldsymbol{H}_{2}\right)$ hold. In addition, assume that

$$
\begin{gathered}
\liminf _{y \rightarrow 0^{+}} \frac{h(t, y(t))}{y}>\lambda_{1}, \quad \text { uniformly on } t \in[0,1] \\
\limsup _{y \rightarrow+\infty} \frac{g(t, y(t))}{y}<\lambda_{1}, \quad \text { uniformly on } t \in[0,1]
\end{gathered}
$$

where $\lambda_{1}$ is the first eigenvalue of $T$ defined by (15); then, problem (1) has at least one positive solution.

Proof. It follows from (37) that there exists $r_{1}>0$ such that

$$
g(t, y(t)) \geq \lambda_{1} y, \quad \forall 0 \leq y \leq r_{1}
$$

Let $y^{*} \in P$ be the positive eigenfunction corresponding to its first eigenvalue $\lambda_{1}=r^{-1}(T)$. Then, $y^{*}=\lambda_{1} T y^{*}$; that is,

$$
\begin{aligned}
& y^{*}(t)=\lambda_{1}\left(T y^{*}\right)(t) \\
& =\lambda_{1} \int_{0}^{1} H(t, s) \phi_{q}\left[\int_{0}^{1} H_{1}(s, \tau) a(\tau) y^{*}(\tau) \mathrm{d} \tau\right] \mathrm{d} s, \\
& \left(y^{*}(t)\right)^{\prime \prime}=\lambda_{1}\left(\left(T y^{*}\right)(t)\right)^{\prime \prime} \\
& =-\lambda_{1} \phi_{q}\left[\int_{0}^{1} H_{1}(t, s) a(s) y(s) \mathrm{d} s\right] \leq 0, \\
& y^{*}(0) \\
& \quad=\lambda_{1} \int_{0}^{1} H(0, s) \phi_{q}\left[\int_{0}^{1} H_{1}(s, \tau) a(\tau) y^{*}(\tau) \mathrm{d} \tau\right] \mathrm{d} s \\
& \quad \geq 0, \\
& y^{*}(1) \\
& \quad=\lambda_{1} \int_{0}^{1} H(1, s) \phi_{q}\left[\int_{0}^{1} H_{1}(s, \tau) a(\tau) y^{*}(\tau) \mathrm{d} \tau\right] \mathrm{d} s \\
& \geq 0 .
\end{aligned}
$$

Hence, $y^{*}(t) \geq 0$, for all $t \in[0,1]$. Then, $y^{*}(t)$ is nonnegative concave on $[0,1]$. Thus, $y^{*} \in P \backslash\{\theta\}$.

Let $\left(T_{2} y\right)(t)=\lambda_{1}(T y)(t), y \in C^{+}[0,1]$. Then, $T_{2}$ : $C^{+}[0,1] \rightarrow C^{+}[0,1]$ is a completely continuous linear operator, and $T_{2}\left(P_{1}\right) \subset P, T_{2} y^{*}=\lambda_{1} T y^{*}=y^{*}$.

For any $y \in \partial B_{r_{1}} \cap P$, it follows from (39) that

$$
\begin{aligned}
& (A y)(t) \\
& \geq \int_{0}^{1} H(t, s) \phi_{q}\left[\int_{0}^{1} H_{1}(s, \tau) a(\tau) h(\tau, y(\tau)) \mathrm{d} \tau\right] \mathrm{d} s \\
& \geq \lambda_{1} \int_{0}^{1} H(t, s) \phi_{q}\left[\int_{0}^{1} H_{1}(s, \tau) a(\tau) y(\tau) \mathrm{d} \tau\right] \mathrm{d} s \\
& =\left(T_{2} y\right)(t), \quad t \in[0,1] .
\end{aligned}
$$

Setting $\Omega(P)=B_{r_{1}} \cap P, L_{1}=L_{3}=T_{2}, L_{2}=I$ denotes the identical operator, and $n=1$, in Lemma 7. We have

$$
i\left(A, B_{r_{1}} \cap P, P\right)=0 \text {. }
$$

It follows from (38) that there exist $r_{2}>r_{1}$ and $0<\sigma<1$, such that

$$
g(t, y(t)) \leq \sigma \lambda_{1} y, \quad \forall y \geq r_{2}
$$

Set $\left(T_{1} y\right)(t)=\sigma \lambda_{1}(T y)(t)$. Then, $T_{1}: C^{+}[0,1] \rightarrow$ $C^{+}[0,1]$ is a completely continuous linear operator, and $T_{1}\left(P_{1}\right) \subset P$. Let $r_{4}>r_{2}>r_{1}$.

Now we prove that $i\left(A, B_{r_{4}} \cap P, P\right)=1$. 
Suppose that there exist $y_{2} \in \partial B_{r_{4}} \cap P, \mu_{0} \geq 1$, such that $A y_{2}=\mu_{0} y_{2}$. Let $e\left(y_{2}\right)=\left\{t \in[0,1] \mid y_{2}(t)>r_{2}\right\}$; then, from (43) we have

$$
\begin{aligned}
& \left(A y_{2}\right)(t) \leq \int_{0}^{1} H(t, s) \\
& \cdot \phi_{q}\left[\int_{0}^{1} H_{1}(s, \tau) a(\tau) g\left(\tau, y_{2}(\tau)\right) \mathrm{d} \tau\right] \mathrm{d} s \\
& =\int_{e\left(y_{2}\right)} H(t, s) \\
& \cdot \phi_{q}\left[\int_{0}^{1} H_{1}(s, \tau) a(\tau) g\left(\tau, y_{2}(\tau)\right) \mathrm{d} \tau\right] \mathrm{d} s \\
& +\int_{[0,1] \backslash e\left(y_{2}\right)} H(t, s) \\
& \cdot \phi_{q}\left[\int_{0}^{1} H_{1}(s, \tau) a(\tau) g\left(\tau, y_{2}(\tau)\right) \mathrm{d} \tau\right] \mathrm{d} s \\
& \quad \leq \sigma \lambda_{1} \int_{0}^{1} H(t, s) \\
& \quad \phi_{q}\left[\int_{0}^{1} H_{1}(s, \tau) a(\tau) y_{2}(\tau) \mathrm{d} \tau\right] \mathrm{d} s \\
& +\phi_{q}^{1} H\left[\int_{0}^{1} H_{1}(s, \tau) a(\tau) g\left(\tau, y_{2}(\tau)\right) \mathrm{d} \tau\right] \mathrm{d} s \\
& \quad(t)+M, \\
& \left.\quad y_{0}\right)
\end{aligned}
$$

where $M=\sup _{y \in \partial B_{r_{2}} \cap P} \int_{0}^{1} H(t, s) \phi_{q}\left[\int_{0}^{1} H_{1}(s, \tau) a(\tau) g(\tau\right.$, $\left.\left.y_{2}(\tau)\right) \mathrm{d} \tau\right] \mathrm{d} s$. Thus, $0 \leq \mu_{0} y_{2}(t)=A y_{2}(t) \leq\left(T_{1} y_{2}\right)(t)+M$, $t \in[0,1]$. Since $T_{1}\left(C^{+}[0,1]\right) \subset C^{+}[0,1]$, we have $0 \leq$ $\left(T_{1}^{j}\left(A y_{2}\right)\right)(t) \leq\left(T_{1}^{j}\left(T_{1} y_{2}+M\right)\right)(t), t \in[0,1]$, for $j=1, \ldots, N-$ 1. It means that $\left\|T_{1}^{j}\left(A y_{2}\right)\right\| \leq\left\|T_{1}^{j}\left(T_{1} y_{2}+M\right)\right\|, j=1, \ldots, N-1$. Let

$$
\varepsilon=\frac{1}{2}\left(1-r\left(T_{1}\right)\right)
$$

For any $y \in C^{+}[0,1]$, denote

$$
\|y\|^{*}=\sum_{i=1}^{N}\left[r\left(T_{1}\right)+\varepsilon\right]^{N-i}\left\|T_{1}^{i-1} y\right\| .
$$

It is easy to see that

$$
\mu_{0}\left\|y_{2}\right\|^{*}<\left[r\left(T_{1}\right)+\frac{3 \varepsilon}{2}\right]\left\|y_{2}\right\|^{*}
$$

Since $\mu_{0} \geq 1$, we have from (47) that $1 \leq r\left(T_{1}\right)+3 \varepsilon / 2$, which contradicts (45). Thus, for any $y \in \partial B_{r_{4}} \cap P, \mu \geq 1$, we have that $A y \neq \mu y$. Thus, from Lemma 6, we know that

$$
i\left(A, B_{r_{4}} \cap P, P\right)=1 .
$$

From (42) and (48), we see that

$$
\begin{aligned}
& i\left(A,\left(B_{r_{4}} \cap P\right) \backslash\left(\bar{B}_{r_{1}} \cap P\right), P\right) \\
& \quad=i\left(A, B_{r_{4}} \cap P, P\right)-i\left(A, B_{r_{1}} \cap P, P\right)=1-0=1 .
\end{aligned}
$$

Then, $A$ has at least one fixed point on $\left(B_{r_{4}} \cap P\right) \backslash\left(\bar{B}_{r_{1}} \cap P\right)$. It means problem (1) has at least one positive solution. The proof is completed.

Corollary 12. Suppose that $\left(\boldsymbol{H}_{1}\right)$ and $\left(\boldsymbol{H}_{2}\right)$ hold. In addition, assume that

$$
\begin{aligned}
0 & \leq g^{\infty}=\limsup _{y \rightarrow+\infty} \frac{g(t, y(t))}{y} \leq \liminf _{y \rightarrow 0^{+}} \frac{g(t, y(t))}{y} \\
& =g_{0} \leq+\infty .
\end{aligned}
$$

Then, for any

$$
\xi \in\left(\frac{\lambda_{1}}{h_{0}}, \frac{\lambda_{1}}{g^{\infty}}\right),
$$

where $\lambda_{1}$ is the first eigenvalue of $T$ defined by (15), problem (35) has at least one positive solution.

Proof. By (51), we obtain

$$
\begin{gathered}
\liminf _{y \rightarrow 0^{+}} \frac{\xi h(t, y(t))}{y}>\lambda_{1}, \\
\limsup _{y \rightarrow+\infty} \frac{\xi g(t, y(t))}{y}<\lambda_{1} .
\end{gathered}
$$

It follows from Theorem 11 that Corollary 12 holds. The proof is completed.

\section{Examples}

Example 1. Consider the existence of positive solutions for the following $p$-Laplacian integral boundary value problems:

$$
\begin{aligned}
\left(\phi_{p}\left(y^{\prime \prime}(t)\right)\right)^{\prime \prime}= & (1+t)(\pi-1) y^{2} \\
& +\frac{5}{2016} \ln (1+y), \quad 0<t<1, \\
y(0)= & y(1)=\int_{0}^{1} b(s) y(s) \mathrm{d} s, \\
y^{\prime \prime}(0)= & y^{\prime \prime}(1) \\
= & \phi_{q}\left(\int_{0}^{1} c(s) \phi_{p}\left(y^{\prime \prime}(s)\right) \mathrm{d} s\right),
\end{aligned}
$$

where $f\left(t, y(t), y^{\prime}(t), y^{\prime \prime}(t), y^{\prime \prime \prime}(t)\right)=(1+t)(\pi-1) y^{2}+$ $(5 / 2016) \ln (1+y) \cdot \phi_{p}(t)=|t|^{p-2} t, p>1, \phi_{q}=\phi_{p}^{-1}, 1 / p+1 / q=$ $1, a \in L^{1}[0,1]$ is symmetric on the interval $[0,1], a(t)=1$ for $t \in[0,1]$ is continuous, and $b, c \in L^{1}[0,1]$ are nonnegative symmetric on $[0,1]$. 
Let $h(t, y)=(5 / 2016) \ln (1+y)+(\pi-1) y^{2}$ and let $g(t, y)=$ $(1+t)(\pi-1) y^{2}+(5 / 2016) \ln (1+y)$. Obviously, $h(t, y) \leq$ $f\left(t, y(t), y^{\prime}(t), y^{\prime \prime}(t), y^{\prime \prime \prime}(t)\right) \leq g(t, y)$, and

$$
\begin{aligned}
& \liminf _{y \rightarrow+\infty} \frac{h(t, y(t))}{y}=+\infty, \quad \text { uniformly on } t \in[0,1], \\
& \limsup _{y \rightarrow 0^{+}} \frac{g(t, y(t))}{y}=\frac{5}{2016}
\end{aligned}
$$

uniformly on $t \in[0,1]$.

All conditions of Theorem 8 are satisfied. Consequently, following from Theorem 8 , we know that the boundary value problem (53) has at least one positive solution.

Example 2. Consider the existence of positive solutions for the following $p$-Laplacian integral boundary value problems:

$$
\begin{aligned}
\left(\phi_{p}\left(y^{\prime \prime}(t)\right)\right)^{\prime \prime} & \\
= & y^{3}+\exp \left\{y(t)-\left|y^{\prime}(t)\right|-\left|y^{\prime \prime \prime}(t)\right|-\left|y^{\prime \prime}(t)\right|\right\} \\
& -1-6 \sin y \\
& -\left|\sin ^{6}\left(y(t)+\left|y^{\prime}(t)\right|+\left|y^{\prime \prime}(t)\right|+\left|y^{\prime \prime \prime}(t)\right|\right)\right| \\
& -\left|\frac{\sin \pi t(1-t)}{\sqrt{\pi t(1-t)}}\right|, \quad 0<t<1, \\
y(0)= & y(1)=\int_{0}^{1} b(s) y(s) \mathrm{d} s, \\
y^{\prime \prime}(0) & =y^{\prime \prime}(1)=\phi_{q}\left(\int_{0}^{1} c(s) \phi_{p}\left(y^{\prime \prime}(s)\right) \mathrm{d} s\right),
\end{aligned}
$$

where $\phi_{p}(t)=|t|^{p-2} t, p>1, \phi_{q}=\phi_{p}^{-1}, 1 / p+1 / q=1$, $a \in L^{1}[0,1]$ is symmetric on the interval $[0,1], a(t)=1$ for $t \in[0,1]$ is continuous, and $b, c \in L^{1}[0,1]$ are nonnegative symmetric on $[0,1]$. For $\left(t, y, y^{\prime}, y^{\prime \prime}, y^{\prime \prime \prime}\right) \in(0,1) \times[0,+\infty) \times$ $(-\infty,+\infty) \times(-\infty,+\infty) \times(-\infty,+\infty)$,

$$
\begin{aligned}
f(t, & \left.y(t), y^{\prime}(t), y^{\prime \prime}(t), y^{\prime \prime \prime}(t)\right) \\
= & y^{3}+\exp \left\{y(t)-\left|y^{\prime}(t)\right|-\left|y^{\prime \prime \prime}(t)\right|-\left|y^{\prime \prime}(t)\right|\right\} \\
& -1-6 \sin y \\
& -\left|\sin ^{6}\left(y(t)+\left|y^{\prime}(t)\right|+\left|y^{\prime \prime}(t)\right|+\left|y^{\prime \prime \prime}(t)\right|\right)\right| \\
& -\left|\frac{\sin \pi t(1-t)}{\sqrt{\pi t(1-t)}}\right|, \quad 0<t<1 .
\end{aligned}
$$

Let

$$
\begin{aligned}
& g(t, y)=y^{3}+e^{y}-1-6 \sin y, \\
& h(t, y)=y^{3}-216-3\left|\frac{\sin \pi t(1-t)}{\sqrt{\pi t(1-t)}}\right|,
\end{aligned}
$$

$$
0<t<1
$$

Obviously, $h(t, y) \leq f\left(t, y(t), y^{\prime}(t), y^{\prime \prime}(t), y^{\prime \prime \prime}(t)\right) \leq g(t$, $y)$, and

$$
\begin{aligned}
& \liminf _{y \rightarrow+\infty} \frac{h(t, y(t))}{y}=+\infty, \quad \text { uniformly on } t \in[0,1], \\
& \limsup _{y \rightarrow 0^{+}} \frac{g(t, y(t))}{y}=-5 \neq \lambda_{1},
\end{aligned}
$$

$\left(\lambda_{1}\right.$ is the first eigenvalue of $T$ ), uniformly on $t \in[0,1]$.

All conditions of Theorem 8 are satisfied. Consequently, following from Theorem 8 , we know that the boundary value problem (55) has at least one positive solution.

\section{Conflict of Interests}

The author declares that she has no competing interests.

\section{Acknowledgments}

The author is very grateful to the editor and the anonymous referee for his/her careful reading of the first draft of the paper and making very valuable comments and helpful suggestions. She would like to express her gratitude to Professor Lishan Liu and Professor R. P. Agarwal for their many valuable comments. The author was supported financially by the Foundation of Shanghai Natural Science (13ZR1430100) and the Foundation of Shanghai Municipal Education Commission (DYL201105) and the NNSF of China (no. 11371095 and no. 2013M541455).

\section{References}

[1] X. Zhang, M. Feng, and W. Ge, "Symmetric positive solutions for $p$-laplacian fourth-order differential equations with integral boundary conditions," Journal of Computational and Applied Mathematics, vol. 222, no. 2, pp. 561-573, 2008.

[2] N. Sergejeva, "The regions of solvability for some three point problem," Mathematical Modelling and Analysis, vol. 18, no. 2, pp. 191-203, 2013.

[3] M. P. Sapagovas and A. D. Stikonas, "On the structure of the spectrum of a differential operator with a nonlocal condition," Differential Equations, vol. 41, no. 7, pp. 1010-1018, 2005.

[4] N. Sergejeva, "On some problems with nonlocal integral condition," Mathematical Modelling and Analysis, vol. 15, no. 1, pp. 113-126, 2010.

[5] J. R. L. Webb and G. Infante, "Non-local boundary value problems of arbitrary order," Journal of the London Mathematical Society, vol. 79, no. 1, pp. 238-258, 2009.

[6] X. Zhang, X. Yang, and W. Ge, "Positive solutions of nth-order impulsive boundary value problems with integral boundary conditions in Banach spaces," Nonlinear Analysis. Theory, Methods \& Applications, vol. 71, no. 12, pp. 5930-5945, 2009.

[7] J. X. Sun and G. W. Zhang, "Positive solutions of singular nonlinear Sturm-Liouville problems," Acta Mathematica Sinica, vol. 48, no. 6, pp. 1095-1104, 2005 (Chinese).

[8] D. J. Guo and V. Lakshmikantham, Nonlinear Problems in Abstract Cones, Academic Press, San Diego, Calif, USA, 1988.

[9] D. J. Guo and J. X. Sun, Nonlinear Integral Equations, Shandong Science and Technology Press, 1987 (Chinese). 


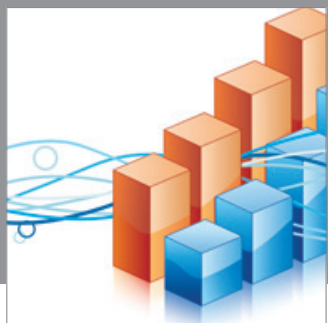

Advances in

Operations Research

mansans

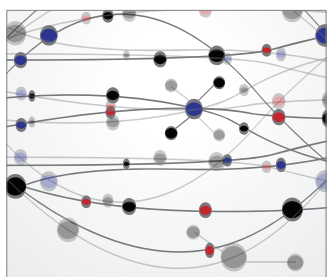

The Scientific World Journal
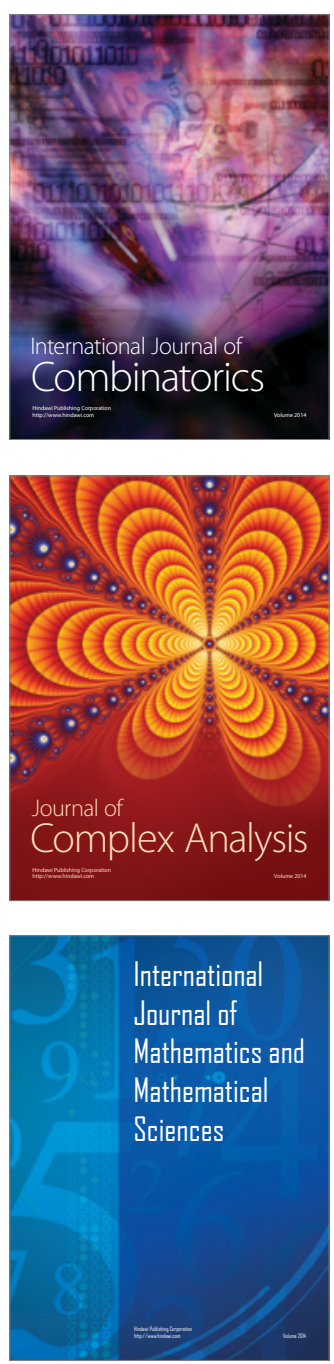
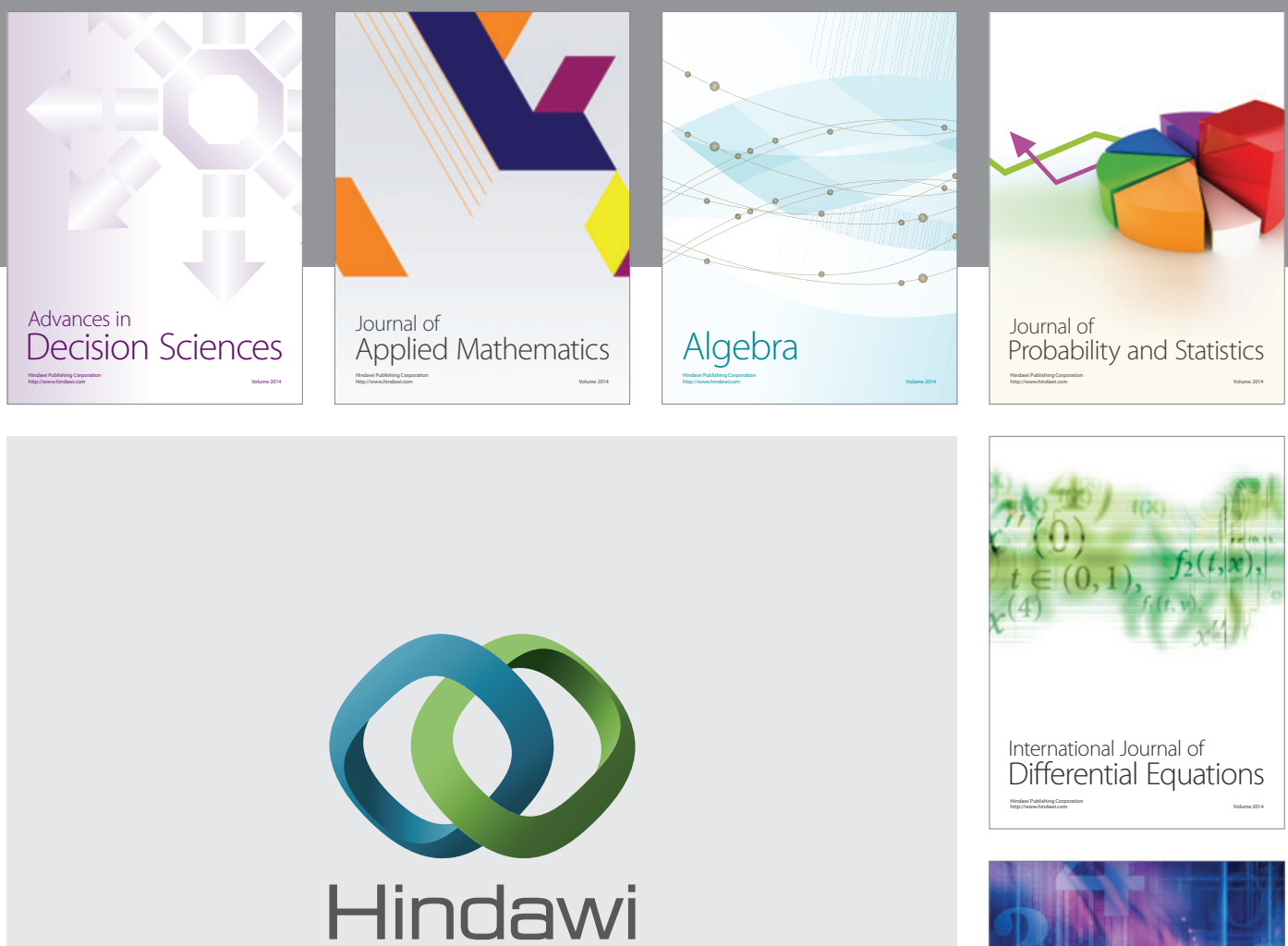

Submit your manuscripts at http://www.hindawi.com
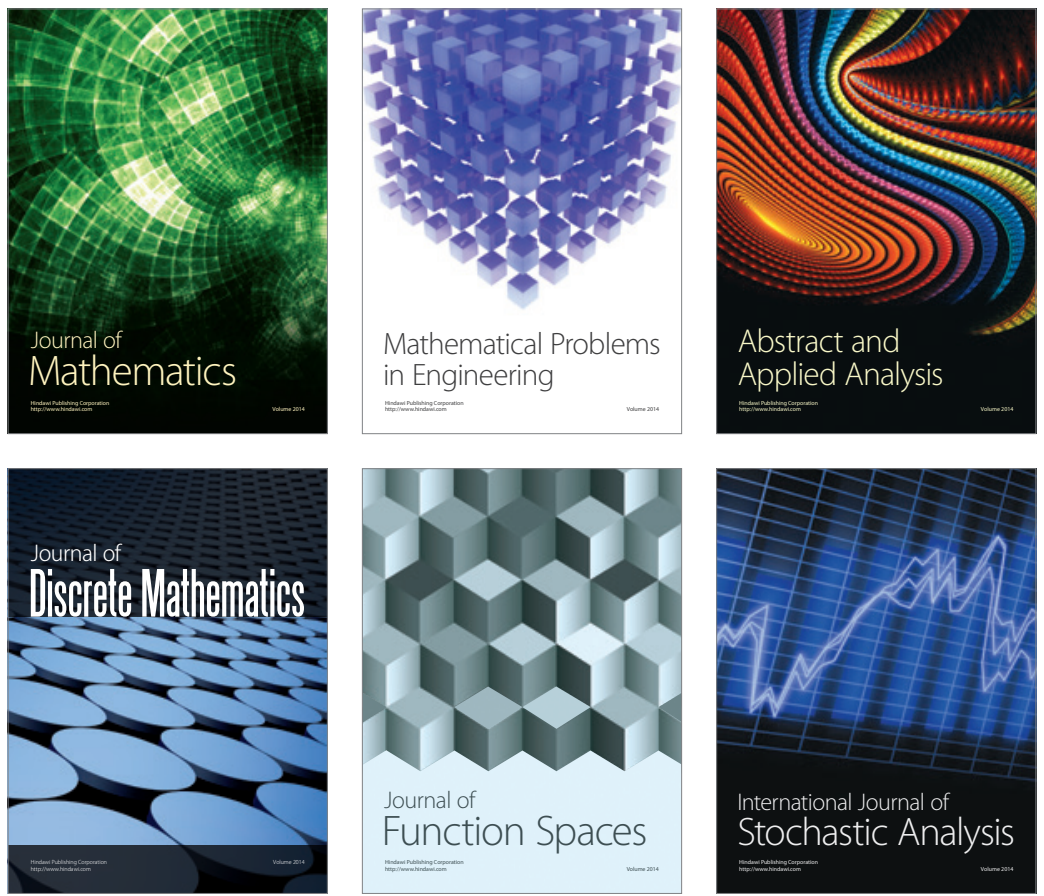

Journal of

Function Spaces

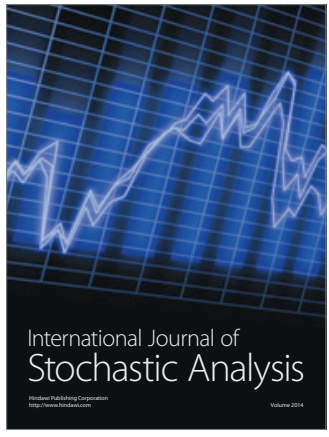

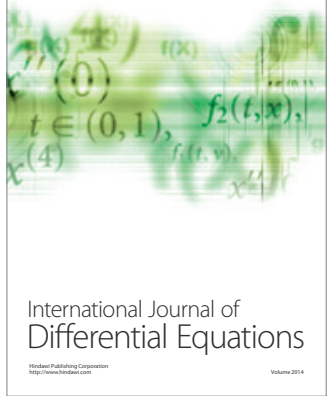
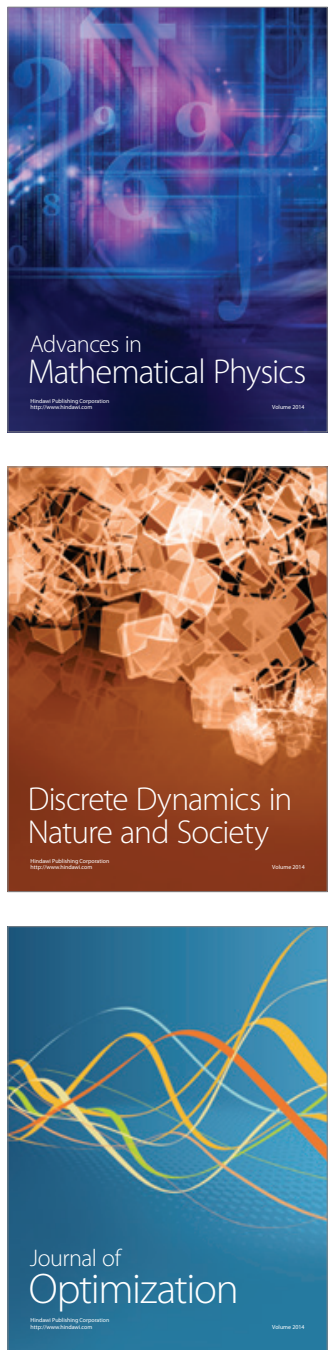